\title{
Article \\ Quantum-Gravitational Trans-Planckian Energy of a Time-Dependent Black Hole
}

\author{
A. J. Nurmagambetov ${ }^{1}$ and I. Y. Park ${ }^{2, *(1)}$ \\ 1 Akhiezer Institute for Theoretical Physics of NSC KIPT, 1 Akademicheskaya St., UA 61108 Kharkov, Ukraine; \\ ajn@kipt.kharkov.ua \\ 2 Department of Applied Mathematics, Philander Smith College, Little Rock, AR 72223, USA \\ * Correspondence: inyongpark05@gmail.com
}

Received: 11 September 2019; Accepted: 11 October 2019; Published: 16 October 2019

\begin{abstract}
We continue our recent endeavor in which a time-dependent black hole solution of a one-loop quantum-corrected Einstein-scalar system was obtained and its near-horizon behavior was analyzed. The energy analysis led to a trans-Planckian scaling behavior near the event horizon. In the present work, the analysis is extended to a rotating black hole solution of an Einstein-Maxwell-scalar system with a Higgs potential. Although the analysis becomes much more complex compared to that of the previous, we observe the same basic features, including the quantum-gravitational trans-Planckian energy near the horizon.
\end{abstract}

Keywords: quantum gravity; loop effects; Firewall; trans-Planckian energy

\section{Introduction}

We are entering an era of multi-messenger astrophysics, and a substantial amount of new data is being collected for various astronomical objects. It is by now firmly established that diverse ultra-high-energy cosmic rays (UHECRs) of extra-galactic origins constantly bombard Earth's atmosphere. Since the energy scale of these particles $-\sim 10^{19} \mathrm{eV}-$ far exceeds that of LHC, study of their origin may well allow us to take a leap in solving some of as-yet unsolved problems in the field.

Although the accumulated data indicate that active galactic nuclei (AGNs) should be largely responsible for the generation of UHECRs, the precise mechanism is yet to be understood. There is wide consensus that the UHECRs must be the work of the super-massive black holes at the centers of the active galaxies. Therefore, the focus of one's quest should be the physics that can produce various extreme-high-energy particles—such as gamma ray photons, protons, heavier ions, and neutrinos-in massive volume. Motivated by this and more theoretically-oriented issues, such as black hole information (see, e.g., [1,2] for reviews) and Firewall [3,4], we have initiated in [5-8] the study of quantum gravitational effects as the potential agent behind certain astrophysical phenomena, including the generation of the UHECR particles.

It is conventionally believed that the quantum gravitational effects are largely negligible. (The same has been believed in the astrophysical situations such as in astrophysical black hole environs.) This view has been challenged through a series of recent works, according to which the quantum-gravitational effects may not only be observable but may also be behind some of the spectacular astrophysical phenomena. In this work, we continue our recent endeavor in which a time-dependent black hole solution of a one-loop quantum-corrected Einstein-scalar system was obtained and its near-horizon behavior was analyzed. The energy analysis led to a trans-Planckian energy behavior near the event horizon, which should lead to observable effects. We extend the analysis to an Einstein-Maxwell-scalar system in the present work. 
To put things in an orderly perspective, let us summarize the status of the matters surrounding the new gravity quantization approach proposed in [9]. A gravity theory can be shown to be one-loop offshell renormalizable in the the conventional covariant quantization framework: for instance, it was shown in the classic paper by 't Hooft and Veltman [10] that pure Einstein gravity is one-loop renormalizable. Once matter fields are included, the one-loop offshell renormalizability is lost. (However, the renormalizability is restored once one includes a cosmological constant that provides more leverage to absorb the one-loop ultraviolet divergences.) At two-loop, things become worse and the conventional offshell renormalizability is lost. Therefore, although one could conduct various quantum-level studies, the nonrenormalizability would force one to stipulate that the results be taken up to the issue of renormalizability, which has been a frustrating setback to further progress. Motivated by the holographical reduction of the physical states [9] (a related discussion can be found in [11]), the covariant quantization has recently been revisited [12]: a way out of the longstanding nonrenormalizability has been proposed, establishing the renormalizability of the physical states that are a certain subset of the perturbative offshell states. Explicit one-loop renormalization procedures have been worked out for several gravity-matter systems [13]. With the new scheme of quantization, one can be assured that the one-loop analysis will remain valid even to higher loops. Further out, the development has provided a stage for further investigation of quantum-gravitational effects and their applications. Through our recent works, it has been shown that the quantum-gravitational effects are of "order-1" in the sense to be reviewed below.

What sets the present work apart from the previous works is that the system being considered is more realistic: we consider an Einstein-Maxwell-scalar system with a Higgs-type potential and its rotating black hole solution. As in [6,7] (see [14] for a related result, where it was observed in a time-dependent setup that the quantum stress-energy tensor inside the black hole reaches a near-Planckian value), the analysis leads to a trans-Planckian energy. The trans-Planckian energy behavior may well be a generic feature of a time-dependent black hole configuration at the quantum level.

The rest of the paper is organized as follows. In Section 2, before embarking on the technical analysis, we give a qualitative reasoning on why there ought to be a trans-Planckian energy behavior near the event horizon. In Section 3, after reviewing the Einstein-scalar system analyzed in [7], we obtain a time-dependent quantum-level solution of an Einstein-Maxwell-scalar system. We consider the $\Lambda_{0}=0$ case-where $\Lambda_{0}$ denotes the classical part of the cosmological constant-for a reason to be explained; extension to the $\Lambda_{0} \neq 0$ case is left for the future. The classical part of the quantum-level solution is required to settle down to a Kerr geometry. A novel feature observed in $[15,16]$ is shared: the quantum effects remove the time-dependence of the classical part, which is crucial for the subsequent energy analysis. In Section 4, we analyze the energy observed near horizon by an infalling observer and are led to a trans-Planckian energy. Although the quantum-induced trans-Planckian energy may sound radical, the result is obtained within the norm of quantum field-theoretic techniques. (Although the framework has radically new ingredients, it is only two- and higher-loop renormalizability that requires such ingredients: the one-loop renormalizability can be established within the conventional framework. The subsequent techniques of finding the time-dependent solution and analyzing the curved space scalar electrodynamics are standard.) Toward the end of Section 4, we briefly comment on the boundary conditions. We reason that the perfect infall boundary condition that is used in the context of the quasi-normal modes is rather restrictive and that more general boundary conditions must be considered to describe the physics of the ring-down phase of a black hole. In Section 5, which is the concluding section, we summarize the results and list future directions. 


\section{Physical Origin of Trans-Planckian Energy}

The present work is motivated in part by the Firewall proposal; let us briefly review the Firewall argument, the backbone of which is as follows. For simplicity, let us take a Schwarzschild black hole. Consider the Kruskal observer and the corresponding vacuum. The Kruskal vacuum must not be a vacuum to a Schwarzschild observer and should appear to be radiating-which is nothing but the Hawking radiation-to a Schwarzschild observer. Now, let us consider things in "reverse": consider a Schwarzschild observer and the corresponding vacuum (or an eigenstate of the observer's number operator). Similar to before, the Schwarzschild vacuum (or the eigenstate) must not be a vacuum to a Kruskal observer. What makes this part of the physics more dramatic is that the Kruskal observer is infalling so the radiation the observer will encounter is highly blue-shifted near the horizon, a Firewall.

One of the goals of the present work (and its sequels) is to back up the Firewall proposal by a quantitative analysis in a more realistic astrophysical environment. What we set out to check in the present work is conceptually simple but highly complicated technically: for one thing, we intend to calculate the quantum-corrected energy measured by an infalling observer near the horizon of a time-dependent rotating black hole of a Einstein-Maxwell-scalar system. To this end, one needs the quantum-corrected action and the field equation with its solution: in particular, a time-dependent solution. Afterwards, one needs to work out the four-velocity of the observer, $U^{\mu}$, in the quantum-corrected background. The stress-energy tensor $T_{\mu v}$ is obtained by taking the functional derivative of the matter part of the action with respect to the metric. We quote the classical part of it here for convenience:

$$
\begin{aligned}
T_{\mu \nu}= & -\frac{2}{\kappa^{2}} \Lambda g_{\mu \nu}+g_{\mu \nu}\left[-\left|\partial_{\rho} \psi-i q A_{\rho} \psi\right|^{2}-m^{2}|\psi|^{2}-\frac{1}{4} F_{\rho \sigma}^{2}\right] \\
& +\left[\left(\partial_{\mu} \psi-i q A_{\mu} \psi\right)\left(\partial_{\nu} \psi^{*}+i q A_{\nu} \psi^{*}\right)+(\mu \leftrightarrow v)\right]+F_{\mu \rho} F_{v}{ }^{\rho}+\mathcal{O}(\hbar),
\end{aligned}
$$

where $g_{\mu v}, A_{\rho}, \psi$ denote the metric, vector field, and scalar, respectively. The energy density $\rho$ measured by the observer is given by $\rho \equiv U^{\mu} U^{v} T_{\mu v}$ where the full quantum-level solution is to be substituted into $T_{\mu \nu}$. Finally, one evaluates the contributions from each term in $T_{\mu \nu}$. As shown below, some of the terms in $\rho$ exhibit a trans-Planckian behavior, which is also an overall behavior of the entire energy density $\rho$.

There is a clear qualitative way to see how the presence of the quantum correction part leads to a trans-Planckian energy (whereas the purely classical analysis does not): when computing the energy density, one may imagine evaluating the right-hand side of the metric field equation, $T_{\mu v}=\frac{G \mu v}{8 \pi G}$, and contract with the four-velocities. The classical metric comes to take the same form as the classical Kerr form that yields a finite energy (zero energy, more precisely). Being an additional contribution to the stress-energy tensor, the presence of the quantum modes changes this status (as we show in more detail below by directly analyzing the stress-energy tensor in Section 4).

One may wonder about the physical origin of such a non-smooth structure in the vicinity of the horizon. Consider a particle heading toward the black hole. It will produce other particles through the quantum-field-theoretic chain reactions on its way to the event horizon. (In the conventional picture, pair creation process has been argued to be responsible for the Hawking radiation. Although the pair-creation process is expected to be one of the main channels of the quantum-gravitational effects, our picture posits a much more complex process than the conventional one where one of the pair particles falls into the black hole while the other one escapes, thereby causing the information loss at the end. When the $\mathrm{BH}$ undergoes an active accretion, such effects must produce a sufficient amount of the outgoing flux for detection. In other words, the quantum-gravitational effects should lead to mass production of cascading particles, some of which escape the black hole.) As an infalling particle accelerates toward the black hole, the acceleration would increase without bound and the particle will release the energy though various radiation channels such as synchrotron radiation and bremsstrahlung. 
One thing worth noting is that the quantum-gravitational effects introduce non-minimal coupling terms such as $\psi \psi^{*} R$. Although the effect of such non-minimal terms is small (and do not play a role in this work), they will lead to violation of the Equivalence Principle. To recap, on the one hand, the quantum gravitational effects are responsible for the trans-Planckian energy, and, on the other hand, they produce such non-minimal couplings, which are at odds with the classical-level understanding of the Equivalence Principle and/or should limit the range of validity thereof.

\section{Time-Dependent Solutions}

Although it is conventionally believed that the quantum gravitational effects are largely negligible, in general, there are circumstances, as our recent works have revealed, in which the quantum-gravitational effects are of "order-1". Such effects may be behind some of the highly energetic astrophysical phenomena. In the previous works [6,7], we considered an Einstein-scalar system and examined the possibility that the quantum corrections may produce a violent energy behavior in the vicinity of the event horizon. It was shown that for a time-dependent solution, one indeed encounters such a behavior. In the present work, we consider an Einstein-Maxwell-scalar system in extension of the previous works.

In Section 3.1, we start by reviewing our earlier work [7] on an Einstein-scalar case. We highlight some salient results of the analysis and point out an undesirable feature of the solution therein obtained. This motivates introduction of a potential in the scalar sector; we consider a Higgs-type potential. In Section 3.2, a time-dependent solution of the Einstein-Maxwell-scalar system with a Higgs potential is obtained. For a reason we detail below, we set the classical part of the cosmological constant to zero, $\Lambda_{0}=0$; extension to $\Lambda_{0} \neq 0$ case will be pursued elsewhere.

\subsection{Review of Einstein-Scalar Case}

Let us warm up by reviewing the case considered in [7], in which an Einstein-scalar system was considered. The classical action in [7] is

$$
S=\frac{1}{\kappa^{2}} \int d^{4} x \sqrt{-g}[R-2 \Lambda]-\int d^{4} x \sqrt{-g}\left[\frac{1}{2}\left(\partial_{\mu} \zeta\right)^{2}+\frac{1}{2} m^{2} \zeta^{2}\right] .
$$

It admits an AdS black hole solution,

$$
d s^{2}=-\frac{1}{z^{2}}\left(F d t^{2}+2 d t d z\right)+\Phi^{2}\left(d x^{2}+d y^{2}\right)
$$

with $\zeta=0$, and

$$
F=-\frac{\Lambda}{3}-2 M z^{3} \quad, \quad \Phi=\frac{1}{z}
$$

The one-loop 1PI effective action is given by [12]

$$
\begin{aligned}
S= & \frac{1}{\kappa^{2}} \int d^{4} x \sqrt{-g}[R-2 \Lambda]-\int d^{4} x \sqrt{-g}\left[\frac{1}{2}\left(\partial_{\mu} \zeta\right)^{2}+\frac{1}{2} m^{2} \zeta^{2}\right] \\
& +\frac{1}{\kappa^{2}} \int d^{4} x \sqrt{-g}\left[e_{1} \kappa^{4} R \zeta^{2}+e_{2} \kappa^{2} R^{2}+e_{3} \kappa^{2} R_{\mu \nu} R^{\mu \nu}+e_{4} \kappa^{6}(\partial \zeta)^{4}+e_{5} \kappa^{6} \zeta^{4}+\cdots\right],
\end{aligned}
$$

where the es are numerical constants that can be determined with the chosen renormalization conditions. The quantum-level field equations can be obtained by varying the action of Equation (5). (As a matter of fact, the boundary conditions must be considered before varying the action. We refer to [13] for potential issues associated with the boundary conditions in varying a gravitational action.) The quantum system admits the following form of the time-dependent metric solution [17]:

$$
d s^{2}=-\frac{1}{z^{2}}\left(F(t, z) d t^{2}+2 d t d z\right)+\Phi^{2}(t, z)\left(d x^{2}+d y^{2}\right)
$$


with the quantum-corrected series

$$
\begin{aligned}
F(t, z) & =F_{0}(t)+F_{1}(t) z+F_{2}(t) z^{2}+F_{3}(t) z^{3}+\ldots \\
& +\kappa^{2}\left[F_{0}^{h}(t)+F_{1}^{h}(t) z+F_{2}^{h}(t) z^{2}+F_{3}^{h}(t) z^{3}+\ldots\right], \\
\Phi(t, z) & =\frac{1}{z}+\Phi_{0}(t)+\Phi_{1}(t) z+\Phi_{2}(t) z^{2}+\Phi_{3}(t) z^{3}+\ldots \\
& +\kappa^{2}\left[\frac{\Phi_{-1}^{h}(t)}{z}+\Phi_{0}^{h}(t)+\Phi_{1}^{h}(t) z+\Phi_{2}^{h}(t) z^{2}+\Phi_{3}^{h}(t) z^{3}+\ldots\right] .
\end{aligned}
$$

Similarly, for the scalar:

$$
\begin{aligned}
\zeta(t, z) & =\zeta_{0}(t)+\zeta_{1}(t) z+\zeta_{2}(t) z^{2}+\zeta_{3}(t) z^{3}+\ldots \\
& +\kappa^{2}\left[\zeta_{0}^{h}(t)+\zeta_{1}^{h}(t) z+\zeta_{2}^{h}(t) z^{2}+\zeta_{3}^{h}(t) z^{3}+\ldots\right]
\end{aligned}
$$

where the modes with superscript " $h$ " represent the quantum modes. The cosmological constant is set to

$$
\Lambda \equiv \Lambda_{0}+\hbar \kappa^{2} \Lambda_{1}
$$

where $\hbar$ has been explicitly displayed for convenience. To solve the scalar and metric field equations, the ansatze above are substituted into the scalar and metric field equations. Then, the resulting expressions are collected in $z$ to the first several orders, and each coefficient is collected in $\hbar$ up to (and including) the first order in $\hbar$. With the help of Wolfram Mathematica package diffgeo.m [18], the order-by-order analysis of the field equations in $z$ and $\hbar$, for the classical modes, leads to

$$
\begin{gathered}
m^{2}=\frac{2 \Lambda_{0}}{3}, \quad \zeta_{0}=0, \quad F_{0}=-\frac{\Lambda_{0}}{3}, \quad \Phi_{1}=0, \quad F_{1}=-F_{0} \Phi_{0}-\Lambda_{0} \Phi_{0} \\
W_{2}=0, \quad F_{2}=F_{0} \Phi_{0}^{2}-2 \partial_{t} \Phi_{0} \\
\zeta_{3}=0, \quad \Phi_{3}=0, \quad F_{3}=\mathrm{const}, \quad \zeta_{4}=0, \quad \Phi_{4}=0, \quad F_{4}=-F_{3} \Phi_{0}
\end{gathered}
$$

and for the quantum modes, to

$$
\begin{gathered}
\zeta_{0}^{h}=0, \quad F_{0}^{h}=-\frac{1}{3} \Lambda_{1}, \quad \Phi_{1}^{h}=0, \quad F_{1}^{h}=\frac{2}{3}\left(3 F_{0}^{h} \Phi_{0}+\Lambda_{0} \Phi_{0} \Phi_{-1}^{h}-\Lambda_{0} \Phi_{0}^{h}-3 \partial_{t} \Phi_{-1}^{h}\right), \\
\Phi_{2}^{h}=0, \quad F_{2}^{h}=\frac{1}{3}\left(-\Lambda_{1} \Phi_{0}^{2}+2 \Lambda_{0} \Phi_{0}^{2} \Phi_{-1}^{h}-2 \Lambda_{0} \Phi_{0} \Phi_{0}^{h}+6 \Phi_{-1}^{h} \partial_{t} \Phi_{0}-6 \partial_{t} \Phi_{0}^{h}\right), \\
\zeta_{3}^{h}=-\frac{1}{\Lambda_{0}}\left(\Lambda_{0} \zeta_{1}^{h} \Phi_{0}^{2}+2 \Lambda_{0} \zeta_{2}^{h} \Phi_{0}+3 \Phi_{0} \partial_{t} \zeta_{1}^{h}+3 \zeta_{1}^{h} \partial_{t} \Phi_{0}+3 \partial_{t} \zeta_{2}^{h}\right), \quad \Phi_{3}^{h}=0, \quad \partial_{t} F_{3}^{h}=-3 F_{3} \partial_{t} \Phi_{-1}^{h}, \\
F_{4}^{h}=F_{3} \Phi_{0} \Phi_{-1}^{h}-F_{3} \Phi_{0}^{h}-F_{3}^{h} \Phi_{0}, \quad \Phi_{4}^{h}=-3 e_{2} F_{3} \Phi_{0}^{2}+3 e_{2} F_{5}-2 e_{3} F_{3} \Phi_{0}^{2}+2 e_{3} F_{5}, \\
\zeta_{4}^{h}=\frac{F_{3} \zeta_{1}^{h}}{2 \Lambda_{0}}+\frac{12 \partial_{t} \zeta_{1}^{h} \partial_{t} \Phi_{0}}{\Lambda_{0}^{2}}+\frac{6 \Phi_{0} \partial_{t}^{2} \zeta_{1}^{h}}{\Lambda_{0}^{2}}+\frac{6 \zeta_{1}^{h} \partial_{t}^{2} \Phi_{0}}{\Lambda_{0}^{2}}+\frac{6 \partial_{t}^{2} \zeta_{2}^{h}}{\Lambda_{0}^{2}}+\frac{9 \Phi_{0}{ }^{2} \partial_{t} \zeta_{1}^{h}}{\Lambda_{0}}+\frac{9 \Phi_{0} \partial_{t} \zeta_{2}^{h}}{\Lambda_{0}}+\frac{9 \zeta_{1}^{h} \Phi_{0} \partial_{t} \Phi_{0}}{\Lambda_{0}} \\
+2 \zeta_{1}^{h} \Phi_{0}^{3}+3 \zeta_{2}^{h} \Phi_{0}^{2} .
\end{gathered}
$$

The subsequent analysis then leads to a trans-Planckian energy for which the quantum modes played a crucial role (see [7] for more details; the corresponding analysis is carried out in Section 4).

As noted in [15], consideration of the field equations at the quantum level leads to additional constraints among some of the classical modes as well, and in particular yields

$$
\zeta_{1}=0=\zeta_{2}
$$

Because these two modes serve as the building blocks of the higher modes, the entire tower of the classical modes comes crumbling down. This shows that the quantum-level field equations deform the classical part as well, although one may naively expect that the classical part will remain 
intact. Many features of the analysis in [7], including the one just mentioned, are present in the Einstein-Maxwell-scalar system, as shown below.

The solution above has an undesirable feature. Because the quantum effects force the entire classical part of the scalar field to vanish, the classical part of the cosmological constant $\Lambda_{0}$ must vanish as well. With $\frac{1}{\Lambda_{0}}$ appearing in some of the mode relationships above, this can potentially be a problem. It turns out that this is not a genuine problem: one can set $\Lambda_{0}=0$ from the beginning. (If one actually sets $\Lambda_{0}=0$ from the beginning, one gets a different solution. We have explicitly checked this although the details are not presented. This means that the classical limit approaches, in the case of a rotating black hole that we consider in the next subsection, the usual Kerr as opposed to the dS / AdS Kerr.) Not unrelated to this, one of the mode relations, $m^{2}=\frac{2 \Lambda_{0}}{3}$, does not look natural. These observations motivate introduction of the scalar potential and, while doing so, we also introduce a Maxwell's field to make the system even more realistic. We come back to these issues in more detail toward the end of the next subsection.

\subsection{Einstein-Maxwell-Scalar System}

With the review of an Einstein-scalar system, we now turn to a more physically relevant system of an Einstein-Maxwell-scalar system with a Higgs potential. The action was given in [13]:

$$
\begin{aligned}
S & =\frac{1}{\kappa^{2}} \int d^{4} x \sqrt{-g}[R-2 \Lambda]+\int d^{4} x \sqrt{-g}\left[c_{1} R^{2}+c_{2} R_{\mu v} R^{\mu v}+\cdots\right] \\
& -\frac{1}{4} \int d^{4} x \sqrt{-g} F_{\mu v} F^{\mu v}-\int d^{4} x \sqrt{-g}\left[\left|\partial_{\mu} \psi-i q A_{\mu} \psi\right|^{2}+\lambda\left(|\psi|^{2}+\frac{1}{2 \lambda} v^{2}\right)^{2}\right] .
\end{aligned}
$$

For our purpose, only the first two leading quantum-correction terms, $c_{1} R^{2}+c_{2} R_{\mu v} R^{\mu \nu}$, are important for the reason explained at the end of this section. Of course, the presence of such terms in the quantum-corrected action can be easily deduced from much older literature. What has been conducted in [13] is explicit Feynman diagrammatic calculations of such terms without pre-determining the forms of such counterterms based on covariance and dimensional analysis. The older literature relies on such an assumption; it has recently been shown (and reviewed in [13]) that explicit establishment of the covariance is a nontrivial task and can be achieved with careful analysis.

The metric and scalar field equations are

$$
\begin{aligned}
& R_{\mu v}-\Lambda g_{\mu v}-\frac{\kappa^{2}}{2} g_{\mu v}\left[\lambda\left(|\psi|^{2}+\frac{1}{2 \lambda} v^{2}\right)^{2}-\frac{1}{4} F_{\alpha \beta} F^{\alpha \beta}\right. \\
& \left.\quad+c_{1} R^{2}+\left(2 c_{1}+c_{2}\right) \nabla^{2} R+c_{2} R_{\alpha \beta} R^{\alpha \beta}+\cdots\right] \\
& +\kappa^{2}\left[-\frac{1}{2}\left(\left(\partial_{\mu} \psi-i q A_{\mu} \psi\right)\left(\partial_{\nu} \psi^{*}+i q A_{v} \psi^{*}\right)+(\mu \leftrightarrow v)\right)-\frac{1}{2} F_{\mu \rho} F_{v}{ }^{\rho}\right. \\
& \left.+2 c_{1} R R_{\mu v}-\left(2 c_{1}+c_{2}\right) \nabla_{\mu} \nabla_{v} R-2 c_{2} R_{\kappa_{1} \mu v \kappa_{2}} R^{\kappa_{1} \kappa_{2}}+c_{2} \nabla^{2} R_{\mu v}+\cdots\right] \\
& =0, \\
& \nabla^{\mu} F_{\mu v}+i q \psi\left(\partial_{v}+i q A_{v}\right) \psi^{*}-i q \psi^{*}\left(\partial_{v}-i q A_{v}\right) \psi+\cdots=0, \\
& \quad\left(\nabla^{\mu}-i q A^{\mu}\right)\left(\nabla_{\mu}-i q A_{\mu}\right) \psi-v^{2} \psi-2 \lambda \psi|\psi|^{2}+\cdots=0 .
\end{aligned}
$$


We find a time-dependent solution by closely following the steps analogous to those reviewed in the previous subsection. Below, we obtain, in a series form, a time-dependent solution that settles down to the standard Kerr geometry (see [19] for review) as the time-dependence fades out. (A similar requirement was imposed in the previously reviewed Einstein-scalar case as well.) It is thus useful to have the following series expansion of the standard Kerr geometry (note that it is a Kerr geometry but not an (A)dS Kerr for a reason to be explained below),

$$
\begin{gathered}
d s^{2}=-\left(1-\frac{2 M z}{1+a^{2} z^{2} \cos ^{2} \theta}\right)\left(d t+a \sin ^{2} \theta d \phi\right)^{2} \\
+2\left(d t+a \sin ^{2} \theta d \phi\right)\left(-\frac{d z}{z^{2}}+a \sin ^{2} \theta d \phi\right)+\left(\frac{1}{z^{2}}+a^{2} \cos ^{2} \theta\right)\left(d \theta^{2}+\sin ^{2} \theta d \phi^{2}\right),
\end{gathered}
$$

where the factor in front of $\left(d t+a \sin ^{2} \theta d \phi\right)^{2}$ can be expanded:

$$
1-\frac{2 M z}{1+a^{2} z^{2} \cos ^{2} \theta}=1-2 M z+2 a^{2} M \cos ^{2} \theta z^{3}-2 a^{4} M \cos ^{4} \theta z^{5}+2 a^{6} M \cos ^{6} \theta z^{7}+\cdots .
$$

For a time-dependent solution, let us try the following ansatz:

$$
\begin{aligned}
d s^{2}= & -\frac{F(t, z, \theta)}{z^{2}}\left(d t+a \sin ^{2} \theta d \phi\right)^{2}+2\left(d t+a \sin ^{2} \theta d \phi\right)\left(-\frac{d z}{z^{2}}+a \sin ^{2} \theta d \phi\right) \\
& +\Phi^{2}(t, z, \theta)\left(d \theta^{2}+\sin ^{2} d \phi^{2}\right) \\
= & -\frac{F(t, z, \theta)}{z^{2}} d t^{2}-\frac{2}{z^{2}} d t d z+2 a\left(-\frac{F(t, z, \theta)}{z^{2}}+1\right) \sin ^{2} \theta d t d \phi-\frac{2 a}{z^{2}} \sin ^{2} \theta d z d \phi \\
& +\Phi^{2}(t, z, \theta) d \theta^{2}+\left(-\frac{a^{2} F(t, z, \theta)}{z^{2}} \sin ^{2} \theta+2 a^{2} \sin ^{2} \theta+\Phi^{2}(t, z, \theta)\right) \sin ^{2} \theta d \phi^{2}
\end{aligned}
$$

For the field variables, let us take the following ansatze: for the scalar field,

$$
\begin{aligned}
\psi(t, z, \theta, \phi) & =\psi_{0}(t, \theta)+\psi_{1}(t, \theta) z+\psi_{2}(t, \theta) z^{2}+\psi_{3}(t, \theta) z^{3}+\ldots \\
& +\kappa^{2}\left[\psi_{0}^{h}(t, \theta)+\psi_{1}^{h}(t, \theta) z+\psi_{2}^{h}(t, \theta) z^{2}+\psi_{3}^{h}(t, \theta) z^{3}+\ldots\right] .
\end{aligned}
$$

For the vector field (this form of the ansatz does not cover the charged black hole case; we leave the charged case for the future investigation),

$$
A_{\mu}(t, z, \theta, \phi)=\left(0, A_{1}(t, z, \theta), A_{2}(t, z, \theta), A_{3}(t, z, \theta)\right)
$$

with

$$
\begin{aligned}
A_{1}(t, z, \theta) & =A_{z 0}(t, \theta)+A_{z 1}(t, \theta) z+A_{z 2}(t, \theta) z^{2}+A_{z 3}(t, \theta) z^{3}+\ldots \\
& +\kappa^{2}\left[A_{z 0}^{h}(t, \theta)+A_{z 1}^{h}(t, \theta) z+A_{z 2}^{h}(t, \theta) z^{2}+A_{z 3}^{h}(t, \theta) z^{3}+\ldots\right], \\
A_{2}(t, z, \theta) & =A_{\theta 0}(t, \theta)+A_{\theta 1}(t, \theta) z+A_{\theta 2}(t, \theta) z^{2}+A_{\theta 3}(t, \theta) z^{3}+\ldots \\
& +\kappa^{2}\left[A_{\theta 0}^{h}(t, \theta)+A_{\theta 1}^{h}(t, \theta) z+A_{\theta 2}^{h}(t, \theta) z^{2}+A_{\theta 3}^{h}(t, \theta) z^{3}+\ldots\right], \\
A_{3}(t, z, \theta) & =A_{\phi 0}(t, \theta)+A_{\phi 1}(t, \theta) z+A_{\phi 2}(t, \theta) z^{2}+A_{\phi 3}(t, \theta) z^{3}+\ldots \\
& +\kappa^{2}\left[A_{\phi 0}^{h}(t, \theta)+A_{\phi 1}^{h}(t, \theta) z+A_{\phi 2}^{h}(t, \theta) z^{2}+A_{\phi 3}^{h}(t, \theta) z^{3}+\ldots\right] .
\end{aligned}
$$


For the metric,

$$
\begin{aligned}
F(t, z, \theta) & =F_{0}(t, \theta)+F_{1}(t, \theta) z+F_{2}(t, \theta) z^{2}+F_{3}(t, \theta) z^{3}+\ldots \\
& +\kappa^{2}\left[F_{0}^{h}(t, \theta)+F_{1}^{h}(t, \theta) z+F_{2}^{h}(t, \theta) z^{2}+F_{3}^{h}(t, \theta) z^{3}+\ldots\right] \\
\Phi(t, z, \theta) & =\frac{1}{z}+\Phi_{0}(t, \theta)+\Phi_{1}(t, \theta) z+\Phi_{2}(t, \theta) z^{2}+\Phi_{3}(t, \theta) z^{3}+\ldots \\
& +\kappa^{2}\left[\frac{\Phi_{-1}^{h}(t, \theta)}{z}+\Phi_{0}^{h}(t, \theta)+\Phi_{1}^{h}(t, \theta) z+\Phi_{2}^{h}(t, \theta) z^{2}+\Phi_{3}^{h}(t, \theta) z^{3}+\ldots\right]
\end{aligned}
$$

where the modes with superscript " $h$ " represent the quantum modes. The quantum corrections of the metric imply a deformation of the geometry by quantum effects [20]. The cosmological constant $\Lambda$ is set to $\Lambda=\Lambda_{0}+\hbar \kappa^{2} \Lambda_{1}$ with vanishing $\Lambda_{0}, \Lambda_{0}=0$, to prevent occurrence of the undesirable feature noted for the Einstein-scalar system. One can consider the first several z-powers. For each $z$-power, expand the coefficients to the first order of $\hbar$. Our analysis yields, with the help of Wolfram Mathematica package diffgeo.m [18], the following results: for the classical modes,

$$
\begin{aligned}
& \psi_{0}(t, \theta)=\psi_{0}, \quad \psi_{0} \psi_{0}^{*}=-\frac{v^{2}}{2 \lambda}, \\
& \psi_{1}(t, \theta)=0, \quad \psi_{2}(t, \theta)=0, \quad \psi_{3}(t, \theta)=0 \\
& A_{z 0}(t, \theta)=0, \quad A_{\theta 0}(t, \theta)=0, \quad A_{\phi 0}(t, \theta)=0 \\
& A_{z 1}(t, \theta)=0, \quad A_{\theta 1}(t, \theta)=0, \quad A_{\phi 1}(t, \theta)=0 \\
& A_{z 2}(t, \theta)=0, \quad A_{\theta 2}(t, \theta)=0, \quad A_{\phi 2}(t, \theta)=0 \\
& A_{\theta 3}(t, \theta)=0, \quad A_{\phi 3}(t, \theta)=0, \quad A_{\phi 4}(t, \theta)=0 \\
& F_{0}(t, \theta)=0, \quad F_{1}(t, \theta)=0, \quad F_{2}(t, \theta)=1 \\
& F_{3}(t, \theta)=-2 M, \quad F_{4}(t, \theta)=0, \\
& \Phi_{-1}(t, \theta)=1, \quad \Phi_{0}(t, \theta)=0, \quad \Phi_{1}(t, \theta)=\frac{1}{2} a^{2} \cos ^{2} \theta \\
& \Phi_{2}(t, \theta)=0, \quad \Phi_{3}(t, \theta)=-\frac{1}{8} a^{4} \cos ^{4} \theta
\end{aligned}
$$


for the quantum modes,

$$
\begin{aligned}
& \partial_{t} \psi_{0}^{h}(t, \theta)=0, \quad \psi_{0}^{h *}(t, \theta)=\frac{v^{2} \psi_{0}^{h}(t, \theta)}{2 \lambda \psi_{0}^{2}}, \quad \psi_{1}^{h *}(t, \theta)=\frac{v^{2} \psi_{1}^{h}(t, \theta)}{2 \lambda \psi_{0}^{2}}, \\
& \partial_{t} \psi_{1}^{h}(t, \theta)=0, \quad \partial_{t} \psi_{2}^{h}(t, \theta)=0, \quad \psi_{2}^{h *}(t, \theta)=\frac{v^{2} \psi_{2}^{h}(t, \theta)}{2 \lambda \psi_{0}^{2}}, \\
& \psi_{3}^{h *}(t, \theta)=\frac{v^{2} \psi_{3}^{h}(t, \theta)}{2 \lambda \psi_{0}^{2}}, \\
& A_{z 0}^{h}(t, \theta)=-\frac{i \psi_{1}^{h}(t, \theta)}{q \psi_{0}}, \quad A_{\theta 0}^{h}(t, \theta)=-\frac{i \partial_{\theta} \psi_{0}^{h}(t, \theta)}{q \psi_{0}}, \quad A_{\phi 0}^{h}(t, \theta)=0, \\
& A_{\theta 1}^{h}(t, \theta)=-\frac{i \partial_{\theta} \psi_{1}^{h}(t, \theta)}{q \psi_{0}}, \quad A_{\phi 1}^{h}(t, \theta)=0, \quad A_{z 1}^{h}(t, \theta)=-\frac{2 i \psi_{2}^{h}(t, \theta)}{q \psi_{0}}, \\
& A_{z 2}^{h}(t, \theta)=-\frac{3 i\left(2 \lambda \psi_{0}^{2} \psi_{3}^{h *}(t, \theta)+v^{2} \psi_{3}^{h}(t, \theta)\right)}{2 v^{2} q \psi_{0}}, \quad A_{\phi 2}^{h}(t, \theta)=0, \\
& A_{\theta 2}^{h}(t, \theta)=-\frac{i\left(v^{2} q^{2} \partial_{\theta} \psi_{2}^{h}(t, \theta)+\lambda \partial_{t} \partial_{\theta} \psi_{1}^{h}(t, \theta)\right)}{v^{2} q^{3} \psi_{0}}, \quad A_{\phi 3}^{h}(t, \theta)=0, \\
& F_{0}^{h}(t, \theta)=-\frac{1}{3} \Lambda_{1}, \quad F_{1}^{h}(t, \theta)=-2 \partial_{t} \Phi_{-1}^{h}, \\
& F_{2}^{h}(t, \theta)=-\frac{5}{3} a^{2} \Lambda_{1} \cos ^{2} \theta+2 \Phi_{-1}^{h}(t, \theta)-2(\cos 2 \theta+2) \csc \theta \sec \theta \partial_{\theta} \Phi_{-1}^{h}(t, \theta), \\
& \partial_{\theta}^{2} \Phi_{-1}^{h}(t, \theta)=\frac{1}{4}\left(-\cot ^{2} \theta\left[3 F_{3}(t, \theta) \partial_{t} \Phi_{-1}^{h}(t, \theta)+a^{2} \Lambda_{1}(\cos 2 \theta+3)\right]\right. \\
& \left.\quad-2\left(\cos _{2} \theta+3\right) \csc \theta \sec \theta \partial_{\theta} \Phi_{-1}^{h}(t, \theta)\right), \\
& \partial_{t} \Phi_{0}^{h}(t, \theta)=-2 \Phi_{-1}^{h}(t, \theta)+2 \cot \theta \partial_{\theta} \Phi_{-1}^{h}(t, \theta), \\
& \partial_{\theta} \Phi_{0}^{h}(t, \theta)=-2 a^{2} \sin \theta \cos \theta \partial_{t} \Phi_{-1}^{h}(t, \theta), \\
& \Phi_{1}^{h}(t, \theta)=-\frac{3}{2} a^{2} \cos ^{2} \theta \Phi_{-1}^{h}(t, \theta), \quad \Phi_{2}^{h}(t, \theta)=-\frac{1}{2} a^{2} \cos { }^{2} \theta \Phi_{0}^{h}(t, \theta), \\
& \Phi_{3}^{h}(t, \theta)=\frac{7}{8} a^{4} \cos ^{4} \theta \Phi_{-1}^{h}(t, \theta) .
\end{aligned}
$$

Several remarks are in order. Although the field equations are more complex and entangled, there exists, as in [7], a robust pattern in the manner in which the mode relationships above are obtained. The lowest $\hbar$ - and $\kappa$-order terms in the action in Equation (13), i.e., the classical action plus $R^{2}, R_{\mu v} R^{\mu v}$ terms, are important in determining the building blocks of the higher modes. Some of the leading $\hbar$-correction parts introduce additional constraints among the classical modes-which is thus of "order-1" effect, and thereby qualitatively change the classical part of the solution. As the higher-order terms (that are not explicitly shown in Equation (13)) are added to the action in Equation (13) and thus to the field equations, their effects are limited to the newly introduced higher modes that are then determined in terms of the lower modes. (This has been explicitly checked but the details are not presented.) Some mode results in Equation (22) deserve specific comments: one particularly novel feature is that, just as in the Einstein-scalar case analyzed in [7], the classical modes of the matter fields (i.e., the scalar field and Maxwell's field) are removed, as can be seen from the vanishing expressions in the classical mode results in Equation (22), by the quantum-level constraints. There are differences as well. One of the differences is that, unlike in [7], in which the mode $\Phi_{0}(t, \theta)$ is not constrained, it is constrained to vanish here. The field equations constrain $F_{3}$ to be a constant; with the requirement that the solution settles down to the usual Kerr geometry, it is determined to be $F_{3}=-2 M$.

The results above have been obtained by setting $\Lambda_{0}$ to $\Lambda_{0}=0$ from the beginning. Let us clarify this. We suspect that the undesirable feature noted in the Einstein-scalar case should be due to the inadequacy of the ansatz in Equation (17) for an (A)dS case. In the literature, the (A)dS Kerr solution is long known in the Boyer-Lindquist coordinates. The first step in proper handling of a time-dependent 
(A)dS Kerr solution should be to write down the ansatz based on the (A)dS Kerr solution in the Eddington-Finkelstein-type coordinates that we have employed (more on this in the Conclusion).

\section{Near-Horizon Dynamics}

The upshot of the previous section is that the essential quantum-level physics can be captured by the action in Equation (13), and the system admits the following time-dependent solution

$$
\begin{gathered}
g_{\mu \nu}=\left(\begin{array}{cccc}
-\frac{F(t, z, \theta)}{z^{2}} & -\frac{1}{z^{2}} & 0 & a\left(1-\frac{F(t, z, \theta)}{z^{2}}\right) \sin ^{2} \theta \\
-\frac{1}{z^{2}} & 0 & 0 & -\frac{a \sin ^{2} \theta}{z^{2}} \\
0 & 0 & \Phi^{2}(t, z, \theta) & 0 \\
a\left(1-\frac{F(t, z, \theta)}{z^{2}}\right) \sin ^{2} \theta & -\frac{a \sin ^{2} \theta}{z^{2}} & 0 & 2 a^{2} \sin ^{4} \theta-\frac{a^{2} F(t, z, \theta) \sin ^{4} \theta}{z^{2}}+\Phi^{2}(t, z, \theta) \sin ^{2} \theta
\end{array}\right), \\
A_{\mu}(t, z, \theta, \phi)=\left(0, A_{1}(t, z, \theta), A_{2}(t, z, \theta), A_{3}(t, z, \theta)\right), \\
\psi(t, z, \theta, \phi)=\psi(t, z, \theta),
\end{gathered}
$$

with each field component expanded in terms of the modes that satisfy the relationships given in Equations (22) and (23). In this section, we complete the rest of the steps of the energy computation.

To compute the energy in the leading order in $\hbar$ and $\kappa$, it suffices to compute only the classical geodesic, a feature shared by the Einstein-scalar system considered in the previous work [7]. Since the time-dependence of the classical part of the solution is removed by the quantum effects, the classical geometry is that of the Kerr. It turns out that it is the boundary modes that are the building blocks of the time-dependence and represent the deformations. They also take a part in the trans-Planckian energy.

In Section 4.1, we review the computation of the geodesic in the Kerr background. In Section 4.2, we consider reexpansion of the solution around the classical location of the event horizon. In the analysis analogous to that in [7], we show that the energy measured by an infalling observer is trans-Planckian. What we called the "horizon quantum modes" in [7] leads to the trans-Planckian energy. In Section 4.3 (which is not part of the mainstream of the paper), we comment on the boundary conditions.

\subsection{Four-Velocity of an Infalling Observer}

One of the ingredients needed to compute the local energy measured by an infalling observer is the four-velocity vector (see, e.g., $[6,7,21]$ ). As shown in the previous section, the time-dependent pieces of the classical part of the quantum-level solution become constrained to vanish: the time-dependent part of the solution for the field equations is only the quantum correction piece. This implies that the classical part of the stress-energy is that of a Kerr geometry. Since the stress-energy tensor vanishes for a Kerr geometry, one can use the geodesic analysis of Kerr spacetime in order to compute the leading quantum-gravitational correction of the energy.

Let us review the geodesic analysis of Kerr spacetime [22]. The metric admits two Killing vectors:

$$
k_{t}^{\mu}=(1,0,0,0), \quad k_{\varphi}^{\mu}=(0,0,0,1),
$$

which leads to two integrals to the geodesic equations: the energy

$$
E=-g_{\mu \nu} k_{t}^{\mu} U^{v}
$$

and angular momentum projection

$$
l=g_{\mu v} k_{\varphi}^{\mu} U^{v}
$$


where $U^{v}$ is the four-velocity $U^{\mu} \equiv \frac{d x^{\mu}}{d \lambda}$ with $\lambda$ being the proper-time parameter along the geodesic. It is normalized according to

$$
g_{\mu \nu} \frac{d x^{\mu}}{d \lambda} \frac{d x^{\nu}}{d \lambda}=-\mu^{2}
$$

The time-like and light-like geodesics correspond to $\mu=1,0$, respectively. One can show that the four-velocity components are given by (the dot represents $\frac{d}{d \lambda}$ )

$$
\begin{gathered}
\dot{\varphi}=\frac{1}{\frac{1}{z^{2}}+a^{2} \cos ^{2} \theta}\left[\left(a E+\frac{l}{\sin ^{2} \theta}\right)-a \triangle^{-1}(P+\sqrt{R})\right] \\
\dot{t}=\frac{1}{\frac{1}{z^{2}}+a^{2} \cos ^{2} \theta}\left[-a\left(l+a E \sin ^{2} \theta\right)+\left(\frac{1}{z^{2}}+a^{2}\right) \triangle^{-1}(P+\sqrt{R})\right] \\
\dot{z}=-\frac{z^{2}}{\frac{1}{z^{2}}+a^{2} \cos ^{2} \theta} \sqrt{R} \\
\dot{\theta}=\frac{1}{\frac{1}{z^{2}}+a^{2} \cos ^{2} \theta} \sqrt{\Theta}
\end{gathered}
$$

with

$$
\begin{gathered}
\triangle=a^{2}+z^{-2}-\frac{2 M}{z}, \\
P=a l+\left(a^{2}+z^{-2}\right) E, \\
\Theta=\mathcal{K}-(l+E a)^{2}-\cos ^{2} \theta\left[a^{2}\left(\mu^{2}-E^{2}\right)+\frac{l_{0}^{2}}{\sin ^{2} \theta}\right], \\
R=P^{2}-\triangle\left(\mathcal{K}+\frac{\mu^{2}}{z^{2}}\right),
\end{gathered}
$$

where $\mathcal{K}$ is another integral of motion called the Carter constant.

\subsection{Trans-Planckian Energy Near Horizon}

In this section, we derive the main result of the present work, Equation (46). What complicates the analysis, as in [7], is the fact that one cannot directly use the results obtained in Equations (22) and (23) for analyzing the energy behavior near the horizon. Instead, one must consider an expansion around the horizon.

The energy density as measured by a free-falling observer is given by

$$
\rho \equiv T_{\mu \nu} U^{\mu} U^{v}
$$

The stress-energy tensor (see [23-26] for reviews on the quantum-level stress tensor) is obtained by taking the functional derivative of the matter part of the action with respect to the metric:

$$
\begin{aligned}
T_{\mu v}= & -\frac{2}{\kappa^{2}} \Lambda g_{\mu v}+g_{\mu v}\left[-\left|\partial_{\rho} \psi-i q A_{\rho} \psi\right|^{2}-\lambda\left(|\psi|^{2}+\frac{1}{2 \lambda} v^{2}\right)^{2}-\frac{1}{4} F_{\rho \sigma}^{2}\right. \\
& \left.+\hbar\left(c_{1} R^{2}-\left(4 c_{1}+c_{2}\right) \nabla^{2} R+c_{2} R_{\rho \sigma} R^{\rho \sigma}\right)+\cdots\right] \\
& +\left[\left(\left(\partial_{\mu} \psi-i q A_{\mu} \psi\right)\left(\partial_{\nu} \psi^{*}+i q A_{\nu} \psi^{*}\right)+(\mu \leftrightarrow v)\right)+F_{\mu \rho} F_{v}{ }^{\rho}\right. \\
& \left.-2 \hbar\left(2 c_{1} R R_{\mu v}-\left(2 c_{1}+c_{2}\right) \nabla_{\mu} \nabla_{\nu} R-2 c_{2} R_{\kappa_{1} \mu \nu \kappa_{2}} R^{\kappa_{1} \kappa_{2}}+c_{2} \nabla^{2} R_{\mu v}\right)+\cdots\right] .
\end{aligned}
$$


For the leading-order energy correction (the leading correction is of second power in $\hbar$ and of inverse second power in $\kappa$, after the $\kappa$-rescaling of the matter fields to be discussed below), one can use the classical form of the stress-energy tensor,

$$
\begin{aligned}
T_{\mu v}= & -\frac{2}{\kappa^{2}} \Lambda g_{\mu v}+g_{\mu v}\left[-\left|\partial_{\rho} \psi-i q A_{\rho} \psi\right|^{2}-\lambda\left(|\psi|^{2}+\frac{1}{2 \lambda} v^{2}\right)^{2}-\frac{1}{4} F_{\rho \sigma}^{2}\right] \\
& +\left[\left(\partial_{\mu} \psi-i q A_{\mu} \psi\right)\left(\partial_{\nu} \psi^{*}+i q A_{\nu} \psi^{*}\right)+(\mu \leftrightarrow v)\right]+F_{\mu \rho} F_{v}{ }^{\rho} .
\end{aligned}
$$

Note that, although the classical form of the stress-energy tensor is used, the full quantum-level solution is to be substituted into the tensor. An additional simplifying feature is that the terms with $g_{\mu \nu}$ are bound by the geodesic normalization, $U_{\mu} U^{\mu}=-\mu^{2}$, and thus unimportant. (The four-velocity reviewed in Section 4.1 was at the classical level and, in particular, so is the normalization condition. However, in anticipation of the full quantum-level normalization condition, we omit the terms with $g_{\mu \nu}$ from the present leading-order energy correction computation.) Thus, the leading-order quantum correction to the classical energy is given by

$$
\rho \sim\left(\left[\left(\partial_{\mu} \psi-i q A_{\mu} \psi\right)\left(\partial_{\nu} \psi^{*}+i q A_{\nu} \psi^{*}\right)+(\mu \leftrightarrow v)\right]+F_{\mu \rho} F_{v}{ }^{\rho}\right) U^{\mu} U^{\nu}
$$

Pole terms-which later yield trans-Planckian scaling-arise from the scalar or vector kinetic term above. (More specifically, the $\partial_{t} \psi \partial_{t} \psi^{*} \dot{t} \dot{t}$ and $F_{t \rho} F_{t} \rho \dot{t} \dot{t}$ terms produce the pole terms as we show explicitly shortly (in fact, $\dot{\varphi}$ has a pole too; however, we focus on $\dot{t}$ ): $\dot{t}$ scales as $\dot{t} \sim \frac{1}{z-z_{E H}}$ where the classical horizon $z_{E H}$ is located at the vanishing of $a^{2}+z^{-2}-2 M z^{-1}$.)

Since the four-velocity has a pole at the classical location of the event horizon $z=z_{E H}$, a more transparent understanding of the behavior of the matter fields near the horizon can be gained by considering reexpansion of the $z$-series solution in

$$
Y \equiv z-z_{E H}
$$

Let us suppose the following form of expansion of the matter fields around $z_{E H}$ below. (At least to the orders analyzed, the time-dependence of the classical parts of the matter fields is absent. In [7], the similar feature was checked to remain true to all orders in $z$, not just to the first several orders. That was done by solving the field equations with the expansion given in Equation (41). Although the corresponding task for the present system turns out to be too involved, we expect that the feature remains true.)

$$
\begin{aligned}
\psi(t, z, \theta)= & \tilde{\psi}_{0}(t, \theta)+\tilde{\psi}_{1}(t, \theta) Y+\tilde{\psi}_{2}(t, \theta) Y^{2}+\tilde{\psi}_{3} Y^{3}+\ldots \\
& +\kappa^{2}\left[\tilde{\psi}_{0}^{h}(t, \theta)+\tilde{\psi}_{1}^{h}(t, \theta) Y+\tilde{\psi}_{2}^{h}(t, \theta) Y^{2}+\tilde{\psi}_{3}^{h} Y^{3}+\ldots\right], \\
A_{1}(t, z, \theta)= & \tilde{A}_{z 0}(t, \theta)+\tilde{A}_{z 1}(t, \theta) Y+\tilde{A}_{z 2}(t, \theta) Y^{2}+\tilde{A}_{z 3}(t, \theta) Y^{3}+\ldots \\
& +\kappa^{2}\left[\tilde{A}_{z 0}^{h}(t, \theta)+\tilde{A}_{z 1}^{h}(t, \theta) Y+\tilde{A}_{z 2}^{h}(t, \theta) Y^{2}+\tilde{A}_{z 3}^{h}(t, \theta) Y^{3}+\ldots\right], \\
A_{2}(t, z, \theta)= & \tilde{A}_{\theta 0}(t, \theta)+\tilde{A}_{\theta 1}(t, \theta) Y+\tilde{A}_{\theta 2}(t, \theta) Y^{2}+\tilde{A}_{\theta 3}(t, \theta) Y^{3}+\ldots \\
& +\kappa^{2}\left[\tilde{A}_{\theta 0}^{h}(t, \theta)+\tilde{A}_{\theta 1}^{h}(t, \theta) Y+\tilde{A}_{\theta 2}^{h}(t, \theta) Y^{2}+\tilde{A}_{\theta 3}^{h}(t, \theta) Y^{3}+\ldots\right], \\
A_{3}(t, z, \theta)= & \tilde{A}_{\phi 0}(t, \theta)+\tilde{A}_{\phi 1}(t, \theta) Y+\tilde{A}_{\phi 2}(t, \theta) Y^{2}+\tilde{A}_{\phi 3}(t, \theta) Y^{3}+\ldots \\
& +\kappa^{2}\left[\tilde{A}_{\phi 0}^{h}(t, \theta)+\tilde{A}_{\phi 1}^{h}(t, \theta) Y+\tilde{A}_{\phi 2}^{h}(t, \theta) Y^{2}+\tilde{A}_{\phi 3}^{h}(t, \theta) Y^{3}+\ldots\right] .
\end{aligned}
$$


The "tilded" modes are given as sums of the original modes. To the orders that we have checked in Section 3, all of the classical modes (except $\psi_{0}$, which is irrelevant for the energy computation) vanish; because of this, the mode expansion above gets simplified to

$$
\begin{aligned}
& \psi(t, z, \theta)=\tilde{\psi}+\kappa^{2}\left[\tilde{\psi}_{0}^{h}(t, \theta)+\tilde{\psi}_{1}^{h}(t, \theta) Y+\tilde{\psi}_{2}^{h}(t, \theta) Y^{2}+\tilde{\psi}_{3}^{h} Y^{3}+\cdots\right], \\
& A_{1}(t, z, \theta)=\kappa^{2}\left[\tilde{A}_{z 0}^{h}(t, \theta)+\tilde{A}_{z 1}^{h}(t, \theta) Y+\tilde{A}_{z 2}^{h}(t, \theta) Y^{2}+\tilde{A}_{z 3}^{h}(t, \theta) Y^{3}+\ldots\right], \\
& A_{2}(t, z, \theta)=\kappa^{2}\left[\tilde{A}_{\theta 0}^{h}(t, \theta)+\tilde{A}_{\theta 1}^{h}(t, \theta) Y+\tilde{A}_{\theta 2}^{h}(t, \theta) Y^{2}+\tilde{A}_{\theta 3}^{h}(t, \theta) Y^{3}+\ldots\right], \\
& A_{3}(t, z, \theta)=\kappa^{2}\left[\tilde{A}_{\phi 0}^{h}(t, \theta)+\tilde{A}_{\phi 1}^{h}(t, \theta) Y+\tilde{A}_{\phi 2}^{h}(t, \theta) Y^{2}+\tilde{A}_{\phi 3}^{h}(t, \theta) Y^{3}+\ldots\right] .
\end{aligned}
$$

Before getting to the final-stage energy analysis, let us note that rescaling of the matter fields is necessary for correct $\kappa$-scaling of various physical quantities, including the energy. The fact that the matter part of the action comes at higher order of $\kappa^{2}$ implies $[7,27]$ that the solution generically takes the form of

$$
\psi=\frac{\xi}{\kappa}, \quad A_{m}=\frac{a_{m}}{\kappa}, \quad m=1,2,3,
$$

where $\xi, a_{m}$ represents the rescaled scalar and vector fields; they will have series expansions-which are similar to those in Equation (41) - in terms of the modes with tildes. In particular, the modes $\left(\tilde{\xi}_{0}^{h}(t, \theta), \tilde{a}_{z 0}^{h}(t, \theta), \tilde{a}_{\theta 0}^{h}(t, \theta), \tilde{a}_{\phi 0}^{h}(t, \theta)\right)$ play an important role in the energy, as we now show. The location of the horizon at the quantum level, $z_{E H}^{h}$ (whose precise determination we do not pursue in the work), take the form of

$$
z_{E H}^{h}=z_{E H}+\mathcal{O}\left(\kappa^{2}\right)
$$

and this implies

$$
\dot{t} \sim \mathcal{O}\left(\kappa^{-2}\right)
$$

at $z=z_{E H}^{h}$. With this scaling, one gets, for the leading behavior of $\rho$,

$$
T_{\mu \nu} U^{\mu} U^{v} \sim \frac{\kappa^{2} f\left(\tilde{\xi}_{0}^{h}, \tilde{a}_{z 0}^{h}, \tilde{a}_{\theta 0}^{h}, \tilde{a}_{\phi 0}^{h}\right)}{\kappa^{4}} \sim \frac{1}{\kappa^{2}},
$$

where $f\left(\tilde{\xi}_{0}^{h}, \tilde{a}_{z 0}^{h}, \tilde{a}_{\theta 0}^{h}, \tilde{a}_{\phi 0}^{h}\right)$ is a quantity that is proportional to $T_{00}$. A direct calculation yields

$$
\begin{gathered}
T_{00}=\frac{1}{\sin ^{2} \theta\left(a^{2} z_{E H}^{2} \cos ^{2} \theta+1\right)}\left(a^{2} z_{E H}^{6} \sin ^{4} \theta\left(\partial_{t} \tilde{a}_{z 0}^{h}\right)^{2}+z_{E H}^{2}\left(\partial_{t} \tilde{a}_{\phi 0}^{h}\right)^{2}\right. \\
+\sin ^{2} \theta\left[z_{E H}^{4}\left(\partial_{t} \tilde{a}_{z 0}^{h}\right)^{2}\left(a^{2} z_{E H}^{2} \cos ^{2} \theta-2 M z_{E H}+1\right)+2 \partial_{t} \tilde{\xi}_{0}^{h *} \partial_{t} \tilde{\xi}_{0}^{h}\left(a^{2} z_{E H}^{2} \cos ^{2} \theta+1\right)\right. \\
\left.\left.+2 a z_{E H}^{4} \partial_{t} \tilde{a}_{z 0}^{h} \partial_{t} \tilde{a}_{\phi 0}^{h}+z_{E H}^{2}\left(\partial_{t} \tilde{a}_{\theta 0}^{h}\right)^{2}\right]\right)+\cdots, \\
T_{33}=2 q^{2} \tilde{\xi}_{0} \tilde{z}_{0}^{*}\left(\tilde{a}_{\phi 0}^{h}\right)^{2}+\frac{z_{E H}^{2}}{a^{2} z_{E H}^{2} \cos ^{2} \theta+1}\left(a^{2} \sin ^{2} \theta\left(\partial_{t} \tilde{a}_{\phi 0}^{h}\right)^{2}+\left(\partial_{\theta} \tilde{a}_{\phi 0}^{h}\right)^{2}\right. \\
-2 \partial_{t} \tilde{a}_{\phi 0}^{h} \tilde{a}_{\phi 1}^{h}\left[a^{2} z_{E H}^{2} \cos ^{2} \theta+a^{2} z_{E H}^{2} \sin ^{2} \theta+1\right] \\
\left.+z_{E H}^{2}\left(\tilde{a}_{\phi 1}^{h}\right)^{2}\left[a^{2} z_{E H}^{2} \cos ^{2} \theta+a^{2} z_{E H}^{2} \sin ^{2} \theta-2 M z_{E H}+1\right]\right) .
\end{gathered}
$$

where the explicitly shown terms represent the expression inside the parentheses in Equation (39). 


\subsection{On the Boundary Conditions}

Lastly, let us comment on the boundary conditions. Although this subsection is not part of the mainstream of the paper, the observation may be useful for some aspects of gravitational wave research. Dirichlet boundary conditions have been widely considered in quantum and gravitational field theories. The recent works show, however, that a complete description of a gravitational system requires extension of the Hilbert space by including other boundary conditions [13]. Let us examine the mode expansions of Equations (18), (20), and (21). The presence of the dynamic boundary modes such as $\left.\Phi_{-1}^{h}(t, \theta), \Phi_{0}^{h}(t, \theta)\right)$ implies that the solution satisfies a certain Neumann-type boundary condition but not a Dirichlet boundary condition. One noteworthy point is that the boundary condition at the asymptotic infinity closely controls what is happening at the event horizon, as one can see by examining the reexpansions in Equation (42) (and those of the metric fields).

In certain circumstances such as a black hole merger, a fixed boundary condition is considered at the horizon. The most widely used one is a perfect-infall boundary condition in the context of the quasi-normal modes. However, the quantum effects obtained in [7] and the present work seem to suggest more general and inclusive boundary conditions as the natural ones at the horizon. The presence of the aforementioned quantum boundary modes will imply that both transmitted and reflected waves will be present. When the time-dependence fades out, the boundary modes die out and a Dirichlet boundary condition naturally arises.

\section{Conclusions}

In this work, we have considered a time-dependent quantum-corrected black hole solution of an Einstein-Maxwell-scalar with a Higgs potential. We have computed the near-horizon energy measured by an infalling observer. The analysis consists of three components: computation of the quantum action (for which we referred to the previous works) and its time-dependent solution, computation of the four-velocity of the observer, and evaluation of the energy after expansion around the location of the horizon. As in the previous works [6,7], a trans-Planckian energy has resulted. Concerning the physical origin of the trans-Planckian energy behavior, the loop effects become important near the horizon.

The power of the previous works and that of the present is the generality of the analyses and the quantitative conclusions drawn from them. In other words, there exists a robust pattern in determination of the higher order modes in terms of the lower ones: the solutions are built out of several lower modes for which only the first two leading quantum correction terms in the action, $R^{2}, R_{\rho \sigma} R^{\rho \sigma}$, are important.

The present work is motivated in part by recent developments in astrophysics, in particular ultra high energy cosmic rays (UHECRs). Although further work is required, the recent observations indicate active galactic nuclei (AGNs) - the central supermassive black holes of active galaxies—as the candidates of the steady sources of the UHECRs. The relatively new paradigm in the field is the view of the black hole at the center of an AGN as a highly efficient engine that converts the gravitational infall energy into outgoing radiation energy. The quantum gravitational effects may well be the mechanism of generating the energy that feeds various outgoing radiation, especially the UHECRs. Being a more realistic system, we expect that the result of the present work will have applications to the physics of $\gamma$ AGNs and UHECRs.

Before embarking on such an enterprise, there are several more urgent and immediate issues to settle. We present them as near-future directions.

An undesirable feature of the solution of the free scalar sector has motivated the introduction of the potential for the scalar sector and consideration of the vanishing classical part of the cosmological constant. Because of the vanishing cosmological constant, our time-dependent solution settles down to the usual Kerr black hole as opposed to a dS/AdS Kerr black hole. To relax the restriction $\Lambda_{0}=0$, one should first consider the (A)dS-Kerr solution in the present Eddington-Finkelstein-type coordinates. The (A)dS-Kerr solution was long known in the Boyer-Lindquist coordinates. It appears that the 
solution has recently been converted in [28] into the Eddington-Finkelstein-type coordinates that we have employed in the present work.

Another relatively urgent direction is a charged black hole case. Taking the temporal gauge, the solution obtained here cannot cover the time-dependent extension of a charged black hole, since the standard charged black hole has a nonzero $A_{0}$ component. It will be of some interest to study how to incorporate the charged case. Presumably a different gauge choice will have to be made.

Still another direction that lies directly ahead of the path of the present work is curved space electrodynamics. The present setup lays necessary foundations for carrying out quantum-gravitational scalar electrodynamics. In particular, it should be possible to compute the quantum-gravitational Poynting vector, which should be useful in comparing with the present and future observations made for astronomical black holes.

Author Contributions: All authors contributed equally to this work. All authors read and approved the final manuscript.

Funding: This research received no external funding.

Conflicts of Interest: The authors declare no conflict of interest.

\section{References}

1. Mathur, S.D. The Information paradox: A Pedagogical introduction. Class. Quant. Grav. 2009, 26, 224001. [CrossRef]

2. Skenderis, K.; Taylor, M. The fuzzball proposal for black holes. Phys. Rep. 2008, 467, 117. [CrossRef]

3. Almheiri, A.; Marolf, D.; Polchinski, J.; Sully, J. Black Holes: Complementarity or Firewalls? J. High Energy Phys. 2013, 1302, 062. [CrossRef]

4. Braunstein, S.L.; Pirandola, S.; Zyczkowski, K. Better Late than Never: Information Retrieval from Black Holes. Phys. Rev. Lett. 2013, 110, 101301. [CrossRef] [PubMed]

5. Park, I.Y. Indication for unsmooth horizon induced by quantum gravity interaction. Eur. Phys. J. C 2014, 74, 3143. [CrossRef]

6. Park, I.Y. Quantum-corrected Geometry of Horizon Vicinity. Fortschr. Phys. 2017, 65, 1700038. [CrossRef]

7. Nurmagambetov, A.J.; Park, I.Y. Quantum-induced trans-Planckian energy near horizon. J. High Energy Phys. 2018, 1805, 167. [CrossRef]

8. Nurmagambetov, A.J.; Park, I.Y. On Firewalls in quantum-corrected General Relativity. arXiv 2019, arXiv:1909.10048.

9. Park, I.Y. Hypersurface foliation approach to renormalization of ADM formulation of gravity. Eur. Phys. J. C 2015, 75, 459. [CrossRef]

10. 't Hooft, G.; Veltman, M.J.G. One loop divergencies in the theory of gravitation. Ann. Poincare Phys. Theor. 1974, 20, 69-94.

11. Hadad, M.; Rosenblum, L. Derivation of Hamilton-like equations on a non-Cauchy hypersurface and their expected connection to quantum gravity theories. arXiv 2019, arXiv:1905.10665.

12. Park, I.Y. One-loop renormalization of a gravity-scalar system. Eur. Phys. J. C 2017, 77, 337. [CrossRef]

13. Park, I. Foliation-Based Approach to Quantum Gravity and Applications to Astrophysics. Universe 2019, 5, 71. [CrossRef]

14. Kawai, H.; Yokokura, Y. A Model of Black Hole Evaporation and 4D Weyl Anomaly. Universe 2017, 3, 51. [CrossRef]

15. Park, I.Y. "Quantum violation" of Dirichlet boundary condition. Phys. Lett. B 2017, 765, 260. [CrossRef]

16. James, F.; Park, I.Y. Quantum Gravitational Effects on the Boundary. Theor. Math. Phys. 2018, 195, 607; [Teor. Mat. Fiz. 2018, 195, 130. [CrossRef]

17. Murata, K.; Kinoshita, S.; Tanahashi, N. Non-equilibrium Condensation Process in a Holographic Superconductor. J. High Energy Phys. 2010, 1007, 050. [CrossRef]

18. Diffgeo.m package for Wolfram Mathematica. Available online: http://people.brandeis.edu/ headrick/ Mathematica/diffgeo.m (accessed on 8 May 2012).

19. Visser, M. The Kerr spacetime: A Brief introduction. arXiv 2007, arXiv:0706.0622.

20. Park, I.Y. On the pattern of black hole information release. Int. J. Mod. Phys. A 2014, 29, 1450047. [CrossRef] 
21. Lowe, D.A.; Thorlacius, L. Pure states and black hole complementarity. Phys. Rev. D 2013, 88, 044012. [CrossRef]

22. Carter, B. Global structure of the Kerr family of gravitational fields. Phys. Rev. 1968, 174, 1559. [CrossRef]

23. Candelas, P. Vacuum Polarization in Schwarzschild Space-Time. Phys. Rev. D 1980, 21, 2185. [CrossRef]

24. Birrell, N.D.; Davies, P.C.W. Quantum Fields in Curved Space; Cambridge University Press: Cambridge, UK, 1982.

25. Frolov, V.P.; Novikov, I.D. Black Hole Physics: Basic Concepts and New Developments; Springer: Berlin, Germany, 1998.

26. Mukhanov, V.F.; Winitzki, S. Introduction to Quantum Effects in Gravity; Cambridge University Press: Cambridge, UK, 2007.

27. Chadburn, S.; Gregory, R. Time dependent black holes and scalar hair. Class. Quant. Grav. 2014, 31, 195006. [CrossRef]

28. Lake, K.; Zannias, T. Global structure of Kerr-de Sitter spacetimes. Phys. Rev. D 2015, 92, 084003. [CrossRef]

(C) 2019 by the authors. Licensee MDPI, Basel, Switzerland. This article is an open access article distributed under the terms and conditions of the Creative Commons Attribution (CC BY) license (http:/ / creativecommons.org/licenses/by/4.0/). 